\title{
Zur Technik der intraarteriellen Injektionen bei Gehirnerkrankungen und zur Anatomie der Arteria vertebralis.
}

\author{
Von Enderlen, Heidelberg und Justi, Marburg.
}

(Mit 7 Abbildungen auf 2 Tafeln.)

In der Münchner med. Wochenschr. 1919, Nr. 23 berichtete $\mathrm{K} \mathbf{n}$ a u e $\mathbf{r}$ über gemeinschaftlich ausgeführte Salvarsaninjektionen in das arterielle System bei progressiver Paralyse. Er hatte die Absicht, das Mittel auf möglichst direktem Wege und möglichst konzentriert in das Gehirn zu bringen.

Zur Ausführung dieses Wunsches standen nun zwei Wege zur Verfügung:

I. die Carotis interna, bzw. communis,

2. die Arteria vertebralis.

Die Arteria meningea media kam aus technischen und praktischen Gründen nicht in Betracht.

Die Freilegung der Carotis interna bereitet das erste und zweite Mal keinerlei Schwierigkeiten; es gelang mir im Laufe der Zeit, mit ganz kleinem Schnitt und ohne jegliche Gefäßunterbindung an sie heranzukommen.

Den zurückgelegten Weg, d. h. die Art der Freilegung brauche ich wohl nicht zu schildern, da er zur Genüge bekannt ist. Bei der Präparation hielten nur die stets vergrößerten, ziemlich weichen Lymphdrüsen in der Nähe der Gefäße auf. Die Carotis interna wurde nur an der beabsichtigten Injektionsstelle von der Adventitia befreit, die beiden Wundlefzen der Außenhaut zum Fixieren des Gefäßes benützt. Eine feine Nadel mit 0,9 proz. Kochsalzlösung gefüllt stand mittels eines kurzen Gummischlauches mit der salvarsangefüllten Spritze in Verbindung. Ohne Schlauch ist es schwierig, die Einführung in die Arterie vorzunehmen; die Nadel liegt auch weniger sicher, und 
Gefäßschädigungen bei unwillkürlichen Bewegungen des Patienten sind nicht ausgeschlossen. Nach der langsam ausgeführten Injektion wurde die abgeschobene Adventitia über den Einstich verzogen und ganz kurze Zeit komprimiert. Es entstand in keinem einzigen Falle, wie nach den Erfahrungen in der Gefäßnaht anzunchmen war, eine irgendwie störende Blutung. Schichtund Hautnaht vollendeten den kurzdauernden Eingriff. Dieser konnte wegen des psychischen Verhaltens des Patienten nur selten in Lokalanästhesie ausgeführt werden; auch die Heilung litt unter ihm. Mehrere Operierte brachten es fertig, den zum Schutze angelegten Stärkebindenverband abzureißen und an der frischen Wunde herumzukratzen. In diesen Fällen mußte wegen Störung im Wundverlauf länger als wünschenswert war mit der folgenden Injektion gewartet werden. Die Wundinfektion erschwerte den zweiten Eingriff an der Carotis interna, den dritten machte sie unter Umständen unmöglich. In solchen Fällen wurde die Carotis communis möglichst nahe der Teilungsstelle frejgelegt; es war dann das Auffinden der Interna meist möglich, dank der Erinne. rung an die vorhergehenden Eingriffe. Im Falle des Mißlingens wurde der Stamm der Communis hoch oben gewählt, die Nadel nach hinten und außen dirigiert, in die "Gegend" der Interna. Ob die Lichtung dieser erreicht wurde, entzog sich der Beurteilung. Bei den später folgenden Einspritzungen mußte man sich mit der Communis begnügen und den Verlust an Material für das Gehirn in Kauf nehmen.

Auch diesem Vorgehen ist im Laufe der Zeit ein Ziel gesetzt. Der Chirurg kann dem Wunsche des Neurologen nach of tmaliger Injektion mit kurzen Pausen nicht entsprechen. Wir diskutierten daher öfter den Weg der direkten Einspritzung, ohne präparatorische Freilegung des Gefäßes. Dieser erschien mir wegen der Gefahr, bei falschem Wege eine Nekrose in der Umgebung der Halsgefäße zu erzeugen, zunächst etwas unheimlich. Am besten gelingt die Injektion, wenn die Arterie gespannt ist. (Kopfteil des. Operationstisches etwas tiefer gestellt.) Man tastet am Innenrande des M. sternocleido-mastoideus die gut pulsierende Carotis in der Höhe des Schildknorpels und sticht mit feiner Nadel langsam in die Tiefe ein, etwas ge ge $n$ die Richtung des Blutstromes. (Bei umgekehrter Führung gerinnt das Blut in der 
Kanüle.) Wenn die Nadel an der Arterienwand angelangt ist, fühlt man gewöhnlich einen Widerstand; sobald sie eingedrungen ist, strömt hellrotes Arterienblut in dicken, rasch folgenden Tropfen aus der äußeren Öffnung heraus. Fühlt man sich nicht ganz sicher, so spritzt man zuerst physiologische Kochsalzlösung ein und läßt dann erst das Salvarsan folgen. Bei richtiger Lage der Nadel geht die Injektion leicht vor sich, fast lęichter als in eine Vene. Nach Entleerung der Spritze wird die Kanüle herausgezogen; einige kleine Blutstropfen entleeren sich darauf aus der Einstichstelle, dann schließt sich diese. Ein kleiner Verband mit Heftpflaster genügt. Knàuer, welcher über 60 Carotisinjektionen ausführte, schreibt, daß er meist kein Narkotikum brauchte. Einige Male spritzte er Chloräthyl auf oder gab Morphium und Skopolamin. Bei sehr unruhigen Kranken dürfte ein kurzdauernder Ätherrausch zu empfehlen sein, um ungeschickte Be: wegungen zu vermeiden, wenn die Kanüle in dem Arterienrohr steckt. Es ist wohl kaum zu bestreiten, daß dieser Modus der Einführung des Mittels einfacher ist als die Freilegung der Arterie. Der Wiederholung, und zwar der öfteren stehen keine Hindernisse im Wege. Auf die direkte Injektion in die Carotis interna muß man dabei freilich verzichten. Obwohl die Methode die Probe an über 60 Ausführungen bei 8 verschiédenen Patienten bestanden hat, kann ich. einige Bedenken nicht unterdrücken:

Verschiebt sich die Nadel bei einer ungeschickten Bewegung des Patienten und gerät sie in das perivaskuläre Gewebe, ohne $\mathrm{da} B$ es bemerkt wird, dann ist mit den Folgen der Salvarsannekrose - die sehr unangenehm sein können -- zu rechnen. Ferner ist es möglich, da $\beta$ das Arterienrohr bei den tastenden Einstichen, besonders bei Atherosklerose, geschädigt wird und sich ein pulsierendes Hämatom entwickelt. Diese Möglichkeit ist nicht am grünen Tisch konstruiert. Als eine Zeitlang die arterielle Anästhesie ausgeführt wurde; wandten wir sie unter anderen Fällen an der A. brachialis an, um eine Handgelenkresek. tion auszuführen. Sie gelang einwandsfrei, der Eingriff war schmerzlos. Etwa I4 Tage später hatte sich an der Einstichstelle ein pulsierender Tumor entwickelt. Ich resezierte ihn und führte die zirkuläre Gefäßnaht aus. Wie vielfach bewiesen, ist die ,ideale Operation“ des Aneurysma auch an der Carotis 
communis (mit oder ohne Plastik aus der Carotis externa) ausführbar, ja sie muß bei den bekannten Gefahren der Lnterbindung gefordert werden; ganz einfach ist sie nicht. Bei den in Rede stehenden Patienten kommt die immerhin mögliche Wundstörung hinzu, auf welche früher hingewiesen wurde.

Der Weg der subkutanen Salvarsaninjektion in die Carotis ist also gangbar, er birgt aber gewisse Gefahren. - Als zweite Eintrittspforte wurde eingangs die Arteria vertebralis genannt.

Es erübrigt sich, auf die gesamte Literatur der Linterbindung des Gefäßes näher einzugehen; ebenso verzichten wir auf die Wiedergabe der Kasuistik. Beide sind in den Arbeiten von $\mathrm{Küttner}$, welcher die Frage wieder in Fluß brachte und in der Arbeit von $\mathrm{Härtel}$ eingehend berücksichtigt.

A nmerkung. Anzufügen ist ein Aneurysma arteriovenosum der $A$. vertebralis und der $V$. jugularis interna von $T$ ietze. Der der Vene angehörende Zwerchsackanteil wurde mit der Vene reseziert. Die abundante Blutung aus dem arteriellen 'Teil des Sackes stand durch Plombierung mit Muskelstücken (nach

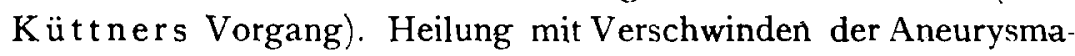
symptome.

Wir haben im Folgenden vorwiegend auf $K$ üt thers und Drüners Mitteilungen einzugehen, und sie hauptsächlich im Hinblick auf unseren $Z$ weck, die Injektion, zu berücksichtigen.

$\mathrm{K} \ddot{\mathrm{u}} \mathrm{t} \mathrm{t}$ e $\mathrm{r}$ unterscheidet bei dem Verlauf der A. vertebralis drei Abschnitte:

I. Die Strecke vom Abgang aus der A. subclavia bis zum Eintritt in das Foramen transversarium des sechsten Halswirbels;

2. diejenige im Verlauf des Canalis transversarius;

3. den in der Regio suboccipitalis gelegenen Abschnitt vom Austritt aus dem Foramen transversarium des Atlas bis zum Eintritt in die Schädelhöhle (Fig. 7). Abweichungen von diesem Schema kommen hin und wieder vor.

Von A nomalien der A. vertebralis möchten wir erwähnen, $\mathrm{da} B$ in einem der Präparate des Marburger Anatomischen Instituts, die wir dem leider verstorbenen Geheimrat $G$ a s s e r verdanken, auf der einen Seite die Art. vertebralis fehlte. Dieser Befund kommt nicht nur für eine beabsichtigte Injektion in Betracht, sondern mahnt, auch 
mit der Unterbindung der A. carotis communis selbst bei jüngeren Individuen nicht allzu freigiebig zu sein.

Einen abnormen Verlauf der A. vertebralis schildert $\mathrm{Kr}$ a u se in H enles Gefäßlehre. Sie trat nicht in das Loch des Querfortsatzes im Epistropheus ein, schlang sich vielmehr in einem nach hinten konvexen Bogen um jenen Fortsatz herum und drang dann in regelmäBiger Weise in das Loch des Querfortsatzes des Atlas ein. In diesem Falle ist das Gefäß einerseits Verletzungen ausgesetzt, andererseits eine ausgiebigere Freilegung möglich. Weiterhin finden wir bei $\mathrm{H}$ enle folgende Vorkommnisse erwähnt: Die A. vertebralis entspringt aus dem Arcus aortae oder der Carotis communis und kann neben dieser außerhalb des Canalis vertebralis verlaufen. Die Arterie tritt in das Foramen transversarium des 7., 5., 4., 3. oder 2. Halswirbels ein und liegt hinter der Carotis communis, (vgl. auch $\mathrm{M}$ on ti und $\mathrm{Wreden}$ ). In der Beobachtung von $\mathrm{L}$ a uenstein ging der Truncus nicht wie normal in gleicher Höhe vor der A. vertebralis ab, sondern $\mathrm{I} 1 / \mathrm{g} \mathrm{cm}$ medial von ihr. La a en $\mathrm{stc}$ in wies ferner darauf hin, daß eine zweite A. vertebralis aus dem Aortenbogen entspringen kann.

In einem Falle von $\mathrm{Hyrtl}$ entsprang die A. vertebralis dextra hinter der Subclavia sinistra und lief in schiefer Richtung hinter der Speiseröhre und vor der Wirbelsäule nach rechts hinüber zum Foramen transversarium des 6. Halswirbels. H y r l erwähnt außerdem, daß die Arteria vertebralis doppclt, selbst dreifach vorkommt, in welchem Falle ihre Wurzeln in verschiedene Querfortsatzlöcher eintreten. Immer vereinigen sich die vervielfältigten Wirbelarterien im Querfortsatzkanal zu einem einfachen Stamm.

$\mathrm{H}$ e yrowsky endlich nimmt an, daB er ein Aneurysma eines ,atypischen größeren Astes" der A. vertebralis operierte.

Indem wir uns der den praktischen Gesichtspunkten entsprechenden Einteilung $K$ üt $t$ ners anschließen, besprechen wir zunächst die topographischen Verhältnissedesersten Abschnittes.

Die Arterie entspringt aus dem aufsteigenden Teile der Subclavia, dicht hinter und lateral vom Truncus thyreo-cervicalis, der sehr verschieden entwickelt ist. In dem vorliegenden Präparat (Fig. I) ist er nur schwach ausgebildet, mit Anomalie des Ursprungs der Arteria thyreoidea inferior und steigt in dem Trigonum scaleno-vertebrale zusammen mit der oben genannten Arterie, und zwar hinter ihr aufwärts zum Querfortsatz des 
sechṣten Halswirbels, dem Tuberculum carotideum. Die: $A$. thyreoidea inferior biegt im obersten Teil des Trigonum niedialwärts $\mathrm{ab}$, um unter der Carotis communis zu der Schilddrüse zu gelangen. Das Trigonum scaleno-vertebrale wird gebildet von dem Scalenus anticus (lateral), dem der-Wirbelsäule aufliegenden longus colli (medial und in der Tiefe). Beide Muskeln heften sich mit partiellen konvergierenden Insertionen am 6 . Halswirbel an. Die Basis des Dreiecks ist die Pleurakuppe (in der Figur grün) und die darauf ruhende Art. subclavia. In unserer Abbildung: ist der longus colli, um die Übersicht nicht zu erschweren, nicht präpariert, sondern ist von der Fascie bedeckt gelassen; siè ist als dunkle Fläche dargestellt. Medial neben den beiden Schlagadern liegt auf dem longus colli der Sympathicus mit seinem mittleren Halsganglion, eingebettet in Faserzüge der Fascia praevertebralis.

Dic Vena vertebralis verläßt den Wirbelkanal nicht selten erst durch das Foramen transversarium des 7. Halswirbels. Sie legt sich gewöhnlich der Arterie von vorne außen an und geht hinter der A. thyreoidea inferior und vor der A. subclavia in die Vena subclavia über.

Die Wirbelschlagader verläuft in erheblicher Tiefe; sie wird bedeckt - abgesehen von der A. thyreoidea inferior und der Vena vertebralis - von der Fascia colli media, dem Sternocleidomastoideus, der $\dot{F}$ ascia colli superficialis und dem Platysma. Bei der Freilegung kommen ferner der M. omohyeoideus und die bald etwas tiefer, bald etwas höher schräg vom Kopfnicker nach hinten und unten herabsteigende Vena jugularis externa in Betracht. Theoretisch kann man auf die A. vertebralis ebensogut vom Vorderrande des Kopfnickers gelangen, indem man die in ein Rohr der Fascia colli media eingehüllten Halsgefäße mit dem Vagus nach hinten zieht, wie von hinten her, indem man den Gefäßstrang nach vorne nimmt.

Was die Operationsverfahren anlangt, so erwähnt $\mathrm{K} u ̈ t \mathrm{tner}$ für die erste Strecke die Verfahren von Fraeys, Maisonneuve, Kocher, Chassaignac (Modifikation Chalot), und Helferich. Küttner selbst ist dafür, bei der Unterbindung im ersten Abschnitt die Durchtrennung des Kopfnickers 
vorzunchmen; entweder der klavikularen oder auch der sternalen Partie. Wir möchten bemerken, daß es sich am Lebenden empfiehlt, außerdem noch die Durchtrennung des M. scalenus anterior hinzuzufügen (cf. Fig. I). Sie wurde von uns bei einer Unterbindung der A. vertebralis nahe dem Abgang aus der A. subclavia zur vollsten Befriedigung ausgeführt. Bei dieser Gelegenheit liel3 sich feststellen, daß die Injektion an dieser Stelle wohl möglich, aber durchaus nicht einfach ist. An eine Wiederholung der Einspritzung auf derselben Seite dürfte kaum ernstlich zu denken sein. Bald nach der oben angeführten Unterbindung empfahl Drüner in einer sehr sorgfältigen Studie über die A. vertebralis ebenfalls die Durchtrennug des Scalenus. $\mathrm{Reh} \mathbf{n}$ resezierte ein Stück der Clavicula und lobt den guten Zugang. In der Giegend des Foramen transversarium legte ich in den Operationkursen das Gefäß zumeist nach Fraeys frei: Ausgiebiger Schnitt am Innenrande des M. sternocleidomastoideus. Nach Spaltung des Platysma und Trennung der Aponeurose zwischen Kopfnicker und Sternothyreoideus Abziehen der großen Gefäße nach außen. In dem lockeren medialen. Bindegewebefilz präsentiert sich bald der Bogen der A. thyreoidea inferior. Vor seiner Mitte etwas nach unten gehend findet man das Tuberculum carotideum und nach Spaltung der Fascie zwischen M. scalenus anterior und longus colli die Arterie. Die partielle Abtragung des Carotidenhöckers (Helferich) gestattet eine weitere Darstellung. Eine Injektion an dieser Stelle vorzunehmen, scheint uns auf Grund unserer Präparationen übersichtlicher zu sein, als wenn man den Weg am hinteren Rande des Kopfnickers wählte (Chassaignac, Oljenick). Joseph fand es ,,durchaus nicht einfach und leicht, zwischen den stark gespannten Sehnenzipfeln des Levator șcapulae und der scaleni bis an die Hinterfläche der Querfortsätze der Halswirbel vorzudringen."

Für den Eingriff am Lebenden möchten wir für die erste Strecke folgenden schon angedeuteten Weg wählen: Hakenförmiger Schnitt in der Höhe des Kehlkopfes beginnend und am Innenrande des Sternocleidomastoideus nach abwärts bis zur Articulatio sterno-clavicularis, und hier kurz oberhalb des Schlüsselbeins parallel mit ihm nach außen abbiegend (cf. Fig. 1). (Küttner und Drüner verlegen den Winkelschnitt nach 
außen und gehen am Hinterrande des Kopfnickers ein. Kü $\mathrm{t} t \mathrm{n}$ e $\mathrm{r}$ gibt jedoch dic Schnittftihrung mit dem Winkel nach innen an als Notbehelf für den Fall, daß zunächst die Freilegung der Carotis interna beabsichtigt war, der weitere Verlauf der Operation ein Aneurysma der Vertebralis ergibt.) Durchtrennung der Kopfnickeransätze mit Rücksicht auf ihre spätere Vereinigung; Durchtrennung des M. omohyoideus und scalenus anterior unter Schonung des $\mathrm{N}$. phrenicus. Wenn möglich, Erhaltung des Kreislaufes der A. transversa colli. Freilegen der A. subclavia und an ihr von lateral nach medial zur Arterie nach Abschieben der Vena vertebralis.

Der Weg im Canalis transversarius (zweiter Abschnitt) kommt für unser Ziel nicht in Betracht. Küttner gab auch für diese Strecke in gewohnter Weise beachtenswerte Winke. $\mathrm{F}$. Krause und Guleke unterbanden die Arterie in dieser Strecke.

Der dritte Abschnitt, nach dem Austritt aus dem Kanal ist für uns wicder zu diskutieren; für Verletzungen kommt er, wie schon Corning in seiner topographischen Anatomie erwähnt, in Betracht. Küt $\mathrm{n}$ er rechnet 40 Proz. dafür heraus. Bei der Skizzierung der to pographis chen Verhältnisse des dritten Abschnittes berücksichtigen wir auch den obersten Teil des Wirbelkanals, weil sich zwischen erstem und zweitem Halswirbel eine verhältnismäßig günstige Stelle für die Freilegung der A. vertebralis bietet. Wie cin Blick auf die Abbildung von $S$ palteholz, die $K$ üt ner in seine Arbeit aufgenommen hat, wie auch unsere Zeichnung 7 lehrt, liegt das Gefäß hier zwar nicht in der Ausdehnung wic im ersten und dritten Abschnitt, jedoch immerhin auf eine für die Operation gut angreifbare Strecke hin frei.

Variationen kommen dabei vor: so ist in unserem Präparat 5 dieser Teil der Arterie sogar länger als der dritte Abschnitt.

An dem Präparat 2 erblicken wir nach Entfernung der Fascie die oberen Enden des Kopfnickers (s. c. m.) vorne, des Trapezius (tr.) hinten, mit annähernd paralleler Faserrichtung. Der vordere Ast des N. accessorius, der den Kopfnicker und den trapezius innerviert, kommt weder jetzt noch beim weiteren Vorgehen in Betracht; der hinter dem Kopfnicker aufsteigende N. occipitalis 
minor kann geschont werden. In der breiten Lücke zwischen den beiden Muskeln ziehen in schräger, mit diesen kontrastierender Richtung dic M. m. splenius capitis (spl.) und levator scapulae (l. sc.) empor. Im oberen hinteren Winkel liegt zwischen Splenius und Trapezius der M. semispinalis capitis (s. sp.) frei. Sein Faserverlauf ist auffällig, weil er sich mit demjenigen der anderen Muskeln kreuzt.

Trägt man nun den Kopfnicker und den dahinter inserierenden Splenius capitis ab und klappt. die Muskeln auseinander (Fig. 3), so erscheinen die tieferen Muskeln. Besonders wichtig für die Orientierung ist der M. longus capitis (l. c.), ein starker plattzylindrischer, oben hin fleischiger Muskel, der das Operationsgebiet gerade von unten nach oben durchzieht. Oben wird er von dem M. obliquus capitis superior (o. c. s.) quer untergriffen. Von dessen Insertion, vorne, am Processus transversus des Atlas, geht im spitzen Winkel der M. obliquus capitis inferior nach hinten (o. c. i.); siehe auch Fig. 7, wo der Muskel durch zwei rote Linien angegeben ist. An gleicher Stelle heftet sich der oberste Zipfel des M. levator scapulae (l. sc.) an (sowie cine sehnige Insertion des M. scalenus medius, die auf der Zeichnung nicht zum Vorschein 'kommt); drängt man den Levator nach vorne, so findet man die Arterie am vorderen Rande des Obliquus capitis inferior zwischen den Processus transversi des Epistropheus (P. t. II) und des Atlas. Quer über die Mitte des Gefäßes legt sich 'der vordere Ast des N. cervicalis II (Fig. 4 und 5). Der N. occipitalis minor bleibt unterhalb des Processus transversus des Epistropheus (Fig. 4).

Rückwärts vom Longus capitis fühlt man am unteren Rande des M. obliquus superior den Processus spinosus des Atlas (Fig. 3 mit einem Kreuz bezeichnet); von da geht die Membrana interspinosa zum Epistropheus hinab (senkrecht schraffiert).

Um an den dritten Abschnitt der Arterie zu gelangen, trägt man die Insertion des Obliquus superior am Processus transversus des Atlas ab und zieht den kräftigen Muskel nach oben. Dabei spannt sich ein Zweig des hinteren Astes des N. cervicalis I an, der sich von unten her um die Arterie, etwa in deren Mitte, herumschlingt (Fig. 5). Das Gefäß beschreibt einen horizontalen, nach außen konvexen Bogen um die Massa lateralis des Atlas, 
wendet sich dann medialwärts, um durch die Membrana atlantooccipitalis in den Wirbelkanal einzutreten. Der N. sympathicus kommt nicht in Frage; ebensowenig der N. accessorius, wie bereits festgestellt.

Venen: Erhebliche Geflechte breiten sich in dem Raum hinter dem Longissimus capitis zwischen Hinterhauptbein und obliquus capitis inferior aus. Sie greifen auch unter den obliquus capitis superior, bedecken also den Zugang zum Abschnitt 3 der Arterie. Wesentlich geringer sind sie am Vorderrande des Longissimus entwickelt, so daß hier die Verletzungsgefahr nicht sehr groß ist (Fig. 6). Für die Unterbindung im dritten Abschnitt fehlte bisher ein typisches Verfahren.

$\mathrm{Küttner}$ füllte diese Lücke aus.

Wir bringen seine Vorschrift:

Der Patient nimmt Bauch- oder Seitenlage ein, mit Drehung des Kopfes nach der gesunden Seite. Längsschnitt von etwa $10 \mathrm{~cm}$ Ausdebnung, vom hinteren Rande des Processus mastoideus senkrecht nach abwärts; mit Vorteil biegt der Schnitt an der Basis des Warzenfortsatzes nach vorne um (mehr Platz). Freilegung des hinteren Kopfnickerrandes, der auf eine kurze Strecke hin abpräpariert nach vorne gezogen wird. Die um den hinteren Rand umbiegenden sensiblen Nerven können meist gut geschont werden. Nun liegt der M. splenius frei. In der Höhe der Spitze des Warzenfortsatzes wird er in einer Länge von $3 \mathrm{~cm}$ quer durchtrennt. Der darunter liegende M. semispinalis wird in gleicher Höhe und Ausdehnung ebenfalls eingeschnitten. Nach Auseinanderziehen der Muskelschnittflächen fühlt man den hinteren Bogen des Atlas; sein oberer scharfer Rand gibt den Anhalt für das Auffinden der Arterie. Zu beachten sind die Venen des Plexus vertebralis posterior. Nun kommt die Freilegung des oberen Randes des Atlasbogens, worauf der N. suboccipitalis erscheint. In der Regel tritt er unterhalb der Arteric aus, kreuzt sie, indem er die Unterbindungsstrecke halbiert. Unter Umständen kann es nötig sein, einen Teil des Atlasbogens abzukneifen.

Anmerkung: Bei einer Stichverletzung der A. vertebralis war ich gezwungen, den Atlasbogen zu resezieren, weil die Gefäßenden sich zurückgezogen hatten. Der Stich hatte die Orientierung etwas gestört, so daß ich ausgedehnter freilegen und die obliqui durchtrennen mußte. Eine. Nervenverletzung konnte vermieden werden. Die Blutung aus dem venösen Plexus war zu beherrschen. Bei dieser Gelegenheit überzeugte ich mich, daß eine sichere Injektion kaum möglich ist. - 
Die Freilegung der Arteric ist nach diesen Angaben an mageren Leichen nicht schwierig; bei muskelkräftigen und fetten Kadavern läßt die Übersichtlichkeit leider zu wünschen übrig.

Wir kommen zum Lebenden. Küttner selbst hat auf die Venen des Plexus vertebralis posterior hingewiesen. F.Krause spricht von den störenden Blutungen des Plexus, ,die in solchen Tiefen viel störender sind als arterielle". Besonders eindringlich geht Drüner auf die Schwierigkeiten der Freilegung im Raum oberhalb des Atlas ein. „Gelangt der Chirurg durch die dicken M. m. splenius und semispinalis in den Spalt hinter dem Atlas, so trifft er gerade da, wo er den Atlasbogen fụhlen, nicht sehen kann, auf die bedenklichste Stelle. Mag er nun stumpf oder scharf vorgehen, ohne Nerven- und Gefäßverletzungen ist es gar nicht möglich, die Arterie zu erreichen, geschweige denn zu unterbinden. Die Blutung in der Tiefe zu stillen, ist sicher nicht immer leicht, stets aber eine langwierige, unangenehme Aufgabe, welche, wenn sie ohne Tamponade gelingt, die Operation aufhält und auch später erschwert. D rüner weist außerdem auf die Gefahren der Luftembolie hin. Bei dem Beugen und Neigen des Kopfes nach der gesunden Seite wird der Raum über dem Atlas bedeutend erweitert, die Venen in ihm können nicht kollabieren, bei bestehendem. negativem Druck von seiten des Sinus. Diese Tatsache müssen wir zugeben, doch können wir ihr nicht die hohe Bedeutung beimessen, welche Drüner ihr zuschreibt.

$\mathrm{Wi}_{\text {ĭ }}$ möchten einige Mitteilungen anführen, welche darauf hinweisen, daß das Aufsuchen der Arterie Schwierigkeiten bereiten kann.

$\mathrm{K}$ at us ch fand die Gegend des dritten Abschnittes sehr blutreich; das Operieren in der Tiefe war sehr erschwert. Er möchte raten, stets einen Querschnitt auf den Küttnerschen Längsschnitt zu legen, oder einen Querschnitt allein zu versuchen. Perthes gelang es nicht, die Arterie obcrhalb des hinteren Bogens des Altas freizulegen. auch dann nicht, als er einen Teil des Randes vom Wirbelbogen abgetragen hatte. (Aneurysma.)

Schemmel schreibt: „Mit vieler Mühe wurde im äuBeren Wundwinkel das Gefäß freigelegt und durch Abtragung des oberen Randes des Atlasbogens zugängig gemacht. Der N. occipitalis major fiel zum Opfer."

Schemmel erwähnt, daß der aufgesetzte Querschnitt nicht 
ganz praktisch war; er hatte über Störungen von seiten des Plexus venosus nicht zu klagen, weder in diesem noch in einem anderen Falle.

Anmerkung: Lauenstein verletzte auf dem $\mathrm{Küt}$ $n$ erschen Wege den Plexus vertebralis posterior mit der Pinzette. Die Blutung, wurde durch zwei angelegte Klemmen beherrscht; wegen der Schwierigkeit der Unterbindung blieben diese liegen. Die Arterie war sodann gut sichtbar.

Wenn es einerseits sicher ist, daß das Freilegen ler Arterie an der eben diskutierten Stelle schwierig sein kann, so ist es andererseits ebenso sicher, daß die Unterbindung daselbst erzwungen werden muß. Auf die unsichere Tamponade (wenn sie auch einige Male Ii etze, Goldammer u. a. glückte), wird man sich nur ungern einlassen. Sitzt die Verletzung oberhalb des Atlas, dann ist bei dem kollateralen. Kreislauf aus den A. a. carotides internae durch Übermittlung der A. basilaris die schönste Linterbindung im ersten Abschnitt zwischen erstem und zweitem Wirbel illusorisch. Ebenso wird man die $\mathrm{K}$ üt $\mathrm{t}$ er sche Stelle wählen, wenn ein Aneurysma sich unterhalb des Atlasbogen entwickelt hat.

Drüner, dessen Bedenken wir oben brachten, schlug vor, die A. vertebralis zwischen dem ersten und zweiten Wirbel im Bereiche des unteren Arterienbogens von der Seite her aufzunehmen. „Dies ist eine kleine, leichte Operation." Die Freilegung zwischen zweitem und drittem Halswibel geht ebenfalls noch gut; zwischen drittem und viertem Wirbel wird die Aufgabe schwieriger, weil die Muskeln zunehmend dicker, dic Querfortsätze größer und der Zwischenraum enger werden.

D r üner empliehlı für die Freilegung der A. vertebralis zwischen erstem und zweitem Wirbel folgende Maßnahmen: Schrïge Seitenlage mit etwas erhöhter Schulter; Kopf nach der anderen Scite gedreht und geneigt. Schnitt von der Mitte des Warzenfortsatzes nach abwärts, zum hinteren Rande des Kopfnickers. Durchschneidung des Sternocleidomastoideus nach oben und vorn, etwa bis zu seiner Mitte, unter Schonung des N. accessorius; der M. splenius wird mit dem longissimus scharf nach hinten gelegt. Unmittelbar unter der Spitze des Warzenfortsatzes fühlt man den Querfortsatz des Atlas; man erkennt die hier entspringenden Bündel des $M$. scalenus medius und des levator scapulae; unter ihm kommt mit gerade nach abwärts gerichtetem Verlauf der sensible Teil des ventralen Astes des zweiten Cervikalnerven. Verfolgt man diesen nach aufwärts unter dem Quer. 
fortsalz des Atlas, indem man die Ursprünge des Ml scalenus medius und des levator scapulae scharf von ihm abliost, so gelangt man in einer Tiefe von etwa $\mathrm{I} 1 / \mathrm{g} \mathrm{cm}$ auf den Strang der Vasa vertebralia, welche vorn und medial unter diesem Nerven kreuzen. Sie haben dabei in der. Lage mit gedrehtem Kopf die Richtung von hinten unten mach vorn oben und sind sowohl uber als unter dem Vervenstamm leicht zugänglich. Störende Blutgefäße sind hier nicht vor. handen. Der Nerv ist mit Sicherheit zu vermeiden und dient als vo:züglicher Leiter zur Auffindung der Arterie. Fï̈r den Fill, dals ess nicht möglich ist, an den unteren Bogen der A. vertebralis heranzukommen (größere Ausdehnung des Aneurysma), macht D rüner zuerst den Schnitt wie zur Enterbindung des unteren bogens. Sudinn: Freilegung des ersten Querfortsatzes unter dem Warzenfortsatz, Querschnitt nach hinten, welcher den Spalt hinter den Stellmuskeln so weit freilegt, daß der dreicckige Schlitz zu erkennen ist. M. splenius, lungissimus und semispinalis werden dabei möglichst nahe am Hinterhaupt durchtrennt und heruntergeklappt. Dann werden dic Muskelansätze am oberen Rande des ersten Querfortsatzes vorsichtig Schicht für Schicht durchtrennt. Es gclingt so, ebenso leicht wie an der Unterseite des ersten Querfortsatzes, an die Arterie heranzukommen. Voraussetzung ist, daß die Vertebralis nicht durch einen Knochenkanal verläuft. Ist dies der Fall, dann muß zuerst der Knochen weggenommen werden.

Wir möchten auf Grund unserer Studien an Leichen folgendes Verfahren vorschlagin:

Iatient liegt auf der Seite, die Schulter unterpolstert, so dals der Kopf nach der gesunden Seite herabhängt; zugleich wird das Kinn gegen die intakte Schulterseite gesenkt. Hautschnitt hinter und oberhalb vom Processus mastoideus beginnend, am hinteren Rande des $M$. sternocleidomastoideus abwärts bis zur Höhe des großen Zungenbeinhorns. Der N. occipitalis minor. der hinter dem Kopfnicker verläuft, kann geschont werden. Der hintere Rand des Kopfnickers wird freigelegt, und der sehnige Ansatz am Warzenfortsatz durchtrennt. Dasselbe geschizht damn mit dem Splenius capitis. Dabej ist zu beachten, daß der unter dem vorderen Rande des Splenius in gleicher Höhe ansetzende Longus capitis, der als Leitmuskel für den weiteren Verlauf der Operation betrachtet werden soll, nicht mit abgelöst wird (Fig.3). Die abgetrennten Muskeln werden stark auseinander gezogen; dadurch legt man sich die tieferen Muskeln frei (Fig. 3). Der N. accessorius liegt im unteren Wundwinkel und kommt nicht 
in Gefahr. Andere Nerwin sind nicht zu berücksichtigen. Der longus capitis wird nach hinter rerzugen, die Ansätz' des Levator scapulae und des scalenus medius am fühlbaren Querforisat. des Atlas abgelöst, die Muskeln werden nach unten geklappt. Nunmehr ist hinreichend Plai geschaffen, um den unteren Raid des M. obliquus capitis inferior freizupräparieren. Hier findet man den rorderen Ast des $\mathrm{N}$. cervicalis II, der die Arterie kreuzt (Fig. 4;. I)iese kimn, wie aus Fig. 5 hervorgeht, auf eine lange Strecke hin bloßgelegt werden, so daß nicht nur eine Unterbindung, sondern auch eine Injektion leicht ausführbar ist. Stärkere Venen treten an dieser Stelle nicht stürend in den Wegr (Fig. 6:.

Will man auf die Arterie in ihrem dritten tbschnitt cindringen, so ist dies auch ausführbar; man hat den M. obliquus capitis superior am Processus transversus des Atlas abzutrennen und aufwärts zu ziehen, wobei sich der hintere Ast des $\mathrm{N}$. cervicalis I, wie bereits oben dargestellt wurde, anspannt (Fig 5 ). Zu beachten sind dabei die starken Venengeflechte (Fig. G), die: durch Blutung recht unangenehm werden können. Über die Luftaspiration (D rü ner) haben wir uns bereits geäußert.

Das Verfahren kann natürlich von vornherein für die Prä. paration des dritten $\Lambda$ bschnites der Arterite eingeschlagen werden. Es gibt eire wesentliche bessere Übersicht als die Methode von $\mathrm{K}$ üt ner. Liegt die Verletzung oder das Aneurysma der A. rertebralis im ersten oder zweiten Abschnitt, so erscheint uns der Raum zwischen zweitem und drittem Halswirbel nach unserem Verfahren als die Stelle der Wahl.

Bezüglich der Injektion, von der wir bei unseren Beobach. tungen ausgingen, konkurriert diese Stelle mit dem ersten Ab. schnitt der Arterie.

\section{Z. usammenfassung.}

Fine Injektion in die das Cehirn versorgenden Arterien kann stattfinden:

I. in die Carotis internal.

a) in das an üblicher Stelle freigelegte Gefäb. Spaltung der Adventitia, Einführung einer feinen, mit kurzem Gummischlauch versehenen und mit Kochsalzlösung gefüllten Nadel. Einspritzung, 
kurzdauernde Kompression nach Reposition der Außenhaut der Arterie. Bei Paralytikern ist gewöhnlich Narkose erforderlich. Störungen des Wundverlaufs, durch unvernünftiges Gebaren der unruhigen Patienten hervorgerufen, erschweren einen weiteren Eingriff, verunmöglichen oft einen dritten. Deshalb wird versucht

b) von der Teilungsitelle der Carotis communis aus die Carotis interna zu präparieren, oder hier die Nadel einzustechen und in die Gegend der Interna zu dirigieren. Die Wiederholungen dieses Eingriffes sind ebenfalls beschränkt;

c) direkte Einspritzung in die durch Tiefliegen des Kopfes angespannte Carotis communis in der Höhe des Ringknorpels. (Je nachdem ist Narkose erforderlich.) Die Nadel wird gegen den Blutstrom eingestochen; zunächst läßt man Kochsalzlösung einlaufen. Mehr als 60 mal bei 8 Patienten ohne Schaden ausgeführt. (Ebenso weitere Injektionen bei anderen Kranken.) Bedenken: Salvarsannekrose in der Lmgebung des Gefäßes, Schädigung der Arterienrohres bei häufiger Applikation (pul. sierendes Hämatom,

2. In die Arteria vertebralis.

Vorkommen von Verlaufsanomalien zu berücksichtigen.

a) Abschnitt I (nach Küttners Einteilung). Die Arterie wird vom Vorderrande des Sternocleidomastoideus unter Abtrennung des Ansatzes dieses Muskels und des Scalenus anticus am Schliisselbein freigelegt;

b) Abschnitt 3 zwischen Atlas und Os occipitale. Fischwerung durch die großen Venenplexus (Methode von $\mathrm{Küt}$ ther);

c) in die Arterie zwischen zweitem und erstem Halswirbel. Fin abgeändertes Freilegungsverfahren gibt freien Überblick über b) und c): Die M. sternocleidomastoideus und splenius capitis werden nach Abtrennung ihrcr Insertion am Schädel aufgeklappt, der M. longus capitis wird als Leitmuskel präpariert. Zwischen ihm und dem vom Processus transversus des Atlas abzutragenden $\Lambda$ nsätzen des Levator scapulae und scalenus medius findet man die Arterie am Vorderrande des M. obliquus capitis inferior; keine erhebliche Störung durch Venenblutung. Der 'Abschnitt 3 kann leicht dargestellt werden durch Abtrennung des M. obliquus capitis superior am Processus transversus des Atlas. 
Zur anatomischen Präparation stellie Herr (ieh. Rat Crasser wie immer ausgesuchtes injiziertes Leichenmaterial zur Verfügung. Herr cand. med. Diehl, Assistent am Anatomischen Institut der Universität Marburg, unterstützte uns in dankenswerter.Weise bei der Herstellung und alem Studium der Präparate.

\section{Tafelerklärung.}

s.chin. $\quad$-. M. Sternocleidomastoideus.

tr. $\quad$-... M. trapezilus.

o. $\quad$ : M. omohyoideus.

sc.a. $\quad \therefore$ M. scalenus anticus.

sc.mi. Mr. scalenus medius.

r.c. ... M. rectus capitis major.

spl. $\quad-$ M. splenius capitis.

b.si. $\quad \cdots$ M. levator scapulac.

s.sj, $\quad$... M. Memispinalis capitis.

l.c. $\quad \therefore$ M. longus capitis.

o.c.s. =- M. obliquus capitis superiur.

o.c.i. $\quad \therefore$ M. obliquus capitis inferior.

bi. $\quad=$ M. biventer mandibulae.

l't. I u. II . Processus transversus des 1. und 2. Halswirbels.

I's. 1 u. II ... I'rocessus spinosus des I. und 2. Halswirbels.

(i. Gelenkspalt zwischen I. und 2. Italswirbel.

M.i. N Membrana interspinosa.

M.u. - . Membrana atlanto-occipitalis.

P. Paukenhöhle.

Ca. $\quad \therefore$ Arteria carotis interna.

A.tr.se : Arteria transversa scapulae.

A.tr.c. Arteria transversa colli.

A.th.. Areria thỵrcoidea inferion.

J.i. $=$ Vena jugularis interna.

J.e. Vena jugularis externa.

V.tr.sc. :- Vená transwersa scapulac.

V.tï.c. = Vena transwersa colli.

O.nii. $\quad-$ Nervus occipitalis nuinor.

O.mi. $\quad \because: N$ Nervus occipitalis major.

C. 3. - Nervus cervicalis III.

N.a.m. :- Nervus auricularis magnus

N.s.c.i. Nervus subcutancus colli inferior.

Dis Zeichnungen sind über den Präparaten auf eine Glasplatte aufgretıagen, danı durchgepaust und in natürlicher Größe ausgeführt. Bei den Figuren $\mathrm{I}-6$ wurde eine Verkleinerung um $1 / 8$ vorgenommen. 
Fig. I: Fensterschnitt: Vorderer Schenkel über dem Sternu-cleido-mastoideus. (s.cl.m.). unterer Schenkel über dem Sr.hlüsselbein. An diesem sieht man den Ansat; des M. pectoralis major. Der Sterno-cleido-mastoideus ist $3 \mathrm{~cm}$ lang an Schlüsselbein abgetrennt und nach vorne ungeschlagen, wodurch die Vena subclavia, Jugularis interna mit der Carotis comm!nnis auf. gederkt werder. Der $M$. omohyoideus $(0)$ ist reseziert, der laterale Stump $f$ nach auß.n um den $M$. trapezius (tr.) geklappt. Der $M$. scalenus anticus (s.c.a.), dessen Ansatz an der ersten Rippe. gerade oberhalb des Schlüsselbeins durch ein nicht schraffiertes Oval angedeutet ist, ist etwas nach hinten gedrängt. Zwischen ihnen und den M. scalenus medius (sc.m.) iiegt der Plexus brachialis (gelb). Dieser ist ein wenig zur Scite geschoben, wodurch der absteigcnde Schenkel der A. subclavia sichtbar wird. Diese ruht auf (ler Pleurakuppe (grün). Aus dem aufsteigenden Schenkel der A. subclavia entspringen: die A. manmaria interna, hinter die V. subclavia hinabtretend, der Truncus thyreocervicalis mit seinen Asten (der A. transversa scapulae (A.tr.sc.), dic oberflächlich vor den $M$. m. scalenus anticus und medius nach der Sehulter hinzieht und unter dem Trapezius verschwindet, der A. intercostalis supremas vor der $V$. subclavia in den Brustkorb verlatufend, und der aufwärtssteigenden A. cervicalis ascendens, bei sc.a. zusammen mit dem $\mathrm{N}$. phrenicus auf dem M. scalenus anticus liegend). (jewöhnlich gibt der Truncus auch die $A$. thyreoidea inferior ab (A.th.i.). In vorliegenden Präparat ist sie cin Ast der cervi. calis ascendenz und ist, wie der Truncus, schwach ausgebildet, was bei stark entwickelter $A$. thyreoidea superior vorkommt; die $A$. thyreoidea inferior kanı auch fehlen. Hinter dem Truncus geht die A. vertebralis ab, dann die A. cervicalis profunda (nach kurzem Verlauf unter den $M$. scalenus anticus untertalichend). $\mathrm{Zu}$ bemerken ist, daß die Ursprungstelle der $\mathrm{A}$. vertebralis wechselt. In der Zeichnungen von $H$ en le liegt sie medial vom Truncus thyreo-cervicalij, ebenso in der Fig. 175 von Corning; dagegen ist sie in Fig. 190 von Corning, ebenso in der Figur von $\mathrm{Spalteholz}$ (bei $\mathrm{K} \ddot{u} \mathrm{t} \mathrm{n}$ er) lateral vom Truncus eingetragen.

Det absteigende Schenkel der $A$. subclavia gibt die $A$. transversa colli (A.tr.c.) ab. Diese liegt in der Regel zunächst auf dem Plexus brachialis, durcbbohrt diesen und verläuft auf dem M. scalenus medius, um unter clem M. trapezius z: verschwinden.

Die Vena vertebralis steigt gewöhnlich, den vorderen außeren Rande der Arterie anliegend, hinter dem $\mathbf{N}$. phrenicus, der $\dot{A}$. cervicalis ascendens und der $A$. thyreoidea inferior hinab in die $V$. subclavia. Zwischen ihr und der V. jugularis interna mündet der Ductus thoracicus (violett) in die V. subclavia ein. Lateral ergießen sich in dic $V$. subclavia die $V$. v. jugularis externa (j.e.), transversa scapulae (V.tr.sc.) und transversa colli (V.tr.c.) ihr Blut. Das Verhalter dieser Venen schwinkt beträchtlich. In dem vorliegenden Iräparat liegt die $V$. jugularis externa ungewöhnlich weit nach außen. Unterhalf, des Ductus thoracicus tritt eine dünne Vene in die Subclavia (V. intercostalis suprema); jhre Einmündungsstelle liegt meist weiter medial (dann $V$. anonyma), bisweilen mehr lateral. 
Dri N. phrenicus geht mit der A. cervicalis schräg abwärts über den Scalenus anticus, an dessen vorderen Rand (in der Zeichnung ist er von diesem abgehoben), und begibt sich, den Truncus thyreo-cervicalis von hinten het umschlingend, unter der $A$. transversa scapulac und in immaria interna an Aer Rückfläche der Vena subclavia in den Brustkorb.

Fig. 2. Fensterschnitt. Vorderer Schenkel vom Processus mastoideus abwärts über den Sterno-cleido-mastoideus bis in die Höhe des großen Zungenbeinhorns; hinterer Schenkel in der hinteren Medianlinie. Im vorderen Teile des Fcnsters der M..sterno-cleido-mastoideus (s.cl.m.); hinten der M. trapezius (tr.) mit seinem Ursprng von der Linea nuchae superior. Beide Muskeln sind längs- und gleichgrerichtet. Der M. splenius capitis (spl.) durchzieht das Fenster schräg nach vorne oben zu seincr Insertion an ler Seitenfläche: und dem hinteren Rande des Warzenfortsatzes, sowie an der Line:a nuchae bis nahe an dic Anheftungsstelle des Trapezius. Vor dem Splenius liegt der M. levator scapulae (l.sc.), der von den vier obersten Halswirbeln entspringt und zu der Basis des sichulterblatts hinabzieht. Hinten oben. in der Lücke zwischen Splenius capitis, Trapezius und Hinterhauptsbasis liegl ein Stück des M. semispinalis capitis (s.sp.) frei. Seine mit den anderen Muskeln kontrastierende Faserung setzt sich zwischen der Linea nuchae superior und inferior an, und zwar unmittelbar unterhalb der Insertion des Trapezius und weiter nach vorne bis zur Insertion des Splenius capitis (unter dem M. obliquus capitis superior).

Fig. 3. Fenstersclnnitt wie in Fig. 2, jedoch nicht so weit nach hinten reichend: der M. trapezius kommt nicht zu Gesicht. Die M. m. sterno-cleidımasteoidcus (s.cl.m.) und splenius capitis (spl.) sind am Ansatz abgetragen und auscinandergeschlagen (der Splenius ist in die Breite ausgezogen, wodurch die Schnittfläche länger erscheint als die Insertionsfläche). Jetzt erscheinen die' ticfercn .Muskeln: am Hinterrande des Kopfnickers der oberste, am Atlas ansetzende Kopf des Levator scapulae (1.sc.), der M. longus capitis (i.c.), senkrecht verlaufend von der Basis des Ilinterhauptbeins zu den Querfortsätzen des dritten bis scchsten Halswirbels. Fr ist etwas nach hinten geschoben; in natürlicher Lage verdeckt er zum Teil dic A. vertebralis uncl den Processus transversus des Epistropheus (P.t.lI). Fierner die M. $\mathrm{m}$. semispinalis capitis (s.sp.), rectus capitis major (r.c.), obliquus capitis superior (o.c.s.) und inferior (o.c.i.). In dem Schlitz zwischen M. m. Jongus capitis, rectus capiti; major, obliquus capitis superior und inferior ist oben der Pruressus spinosus des Atlas (weiß mit Krcuz), darunter die Membrana int( $\cdot$ spinosa zwischen erstem und zweitem Halswirbel (siche auch fï. 7), sunkrecht schraffiert, sichtbar. Oberhalb des M. oblicuus capitis superior ist die: Membraná atlanto-occipitalis (M.o.) angedeutet.

Die A. vertebralis liegt am vorderen Rande des $M$. obliquus capitis inferior (siehe auch Fig. 7). Sie tritt aus dem Foramen transversarium des Epistropheus oberhalb des Processus transversus (P.tr. II) hervor. In djesem Präparat, sowie in den Fig. 4 und 5 ist der rückwärts konvexe Bogen der Arterie durch dic starke Senkung des Kopfes nach der entgegengesetzten 
Seite ausgeglichen. Außerdem steigt die Schlagader nicht wie in Fig. 7 schıäg nach hinten oben, sondern annähernd senkrecht aufwärts, eine Folgt' der Drehung des Kopfes nach der gesunden Seite, wodurch das Forameil transversarium des Atlas nach vorne, senkrecht über dasjenige des Epi stropheus, gelangt.

Fig. 4. Schnitt über den Processus mastoideus, entlang dem Hinter rande des Kopfnickers (s.cl.m.) bis etwas unterhalb des Kieferwinkels. Die Hautuänder sind auseinandergezogen; der Kopfnicker nach vorne genommen. Der M. levator scapulae (l.sc.), die M. m. semispinalis capitis (s.sp.) und sple. nius capitis (spl.) sind nach binten gedrängt; es erscheint in der Tiefe die A. vertebralis, vom Austritt aus dem Foramen transversarium des Epistro pheus (oberhalb des hügelförmigen Processus transversus) bis zu der Stelle, wo sie unter den $M$. obliquus capitis inferior untertaucht, um in das Foramen transversarium des Atlas einzutreten. Die Arterie wird überquert von der. vordcren Ast des $N$. cervicalis. II. Unten verläuft schräg über die $\mathbf{M} . \mathbf{m}$. levator scapulae, M. m. splenius und semispinalis der $N$. accipitalis minor (o.mi.) aus dem dritten Cervicalnerven.

Fig. 5. Fensterschnitt, vom vorderen Rande des Processus mastoideus, entlang dẹm Kopfnicker, bis zur Höhe des Unterkieferwinkels, ninterer Rand nahe der Medianlinie.

M. m. sterno-cleido-mastoideus (s.cl.m.) und splenius apitis (spl) sind am Ansatz abgetrennt und auseinandergeklappt. Ler M. longus capitis (1.c.), der Scalenus medius (sc.m.) und zwei Köpfe des M. levator scapulae. (l.sc.) sind an ihren Insertionen abgetragen und reseziert; der untere Stumpf des $\mathbf{M}$. Iongus capitis und eines Kopfes des Levator scapulae (l.sc.) sind nach unten geschlagell.

Der M. obliquus capitis superior (o.c.s.) ist am Processus cransversarius des Atlas (o.c.s'.) abgeschnitten und nach oben gelegt. Durch die Präparation ist die A. vertebralis vom Foramen transversarium des Epistropheus bis zum Eintritt in den Wirbelkanal sichtbar gemacht, bis auf das vom $M$. obliquus capitis inferior verdeckte. Gebiet des Foramen transversarium des Atlas. Zwei Äste der Arterie sind dargestellt: ein Ramus muscularis (oben) und ein Ra. mus meningeus am unteren Rande des Obliquus capitis inferior, der in das Foramen intervertebrale I eintritt.

Der Abschnitt 3 der Arterie wird von einem Zweig des hinteren Astes des $N$. cervicali; I überkreuzt; dieser hintere Ast tritt unter der Arterie her vor. Der vordere stärkere Ast, sowie der Austritt des Nerven aus dem Wirbel kanal sind durch die Arterie verdeckt. Unter dem M. obliquus capitis in. ferior erscheint, nachdem er das Foramen intervertebrale I verlassen hat, der $\aleph$. cervicalis II, teilt sich dann in einen vorderen und einen stärkeren hinteren Ast. Aus dem hinteren Ast, der sich um den M. obliquus capitis inferior von unten her schlingt, geht außer kleinen Muskelzweigen der $\mathrm{N}$. occipitalis major (o.ma.) hervor. Der vordere Ast überkreuzt dic Arterie in der Mitte zwischen erstem und zweitem Foramen intertransversarium und den Gelenkspalt zwischen Atlas und Epistropheus. (G.; bei dem Kreuz ist eu 
Stück Kapselband dargestellt.) Am Hinterrande des Kopfnickers erscheint die Vena jugularis interna. Hinter ihr tritt der $N$. cervicalis III hervor, der mit dem $N$. cervicalis $I I$ in Verbindung stcht, er gibt nach hinten den $N$ occipitalis minor (o.mi.) ab. (Vgl. auch Fig. 4.)

Fig. 6. Venen mit Berlinerblau injiziert. Vensterschntt. Vorderer Schenkel vom Processus mastoidcus, entlang dem Kopfnicker bis i cm oberhalb des Schlüsselbeins; hinterer Schenkel in der Nähe der hinteren Median linic. Präparation entsprechend der Fig. 3. Auf dem Sterno-cleido-mastoideus die $V$. jugularis externa, an binteren Rande die $V$. jugularis interna. Hinte ihr tritt der $N$ cervicals II hervor mit seinem oberen Ast, dem $N$. auricu laris magnus ( $\mathrm{N}$.a.m.) (geht auf die Vorderfläche des Kopfnickers) und dem N. subcutancus colli inferior (N.s.c.i.). Der N. auricularis magnus hommt bei der Freilegung des obersten Abschnittes der $A$. vertebralis also nicht unter das Messet.

I.sc. =- levator scapulae; sc.m. : scalenus medius; $1 . c$.:- longus capitis; spl $=$ splenius capitis; $\mathrm{bi}=$ biventer mandibulac; o.c.s. und o.c. $\mathrm{i}=$ =bliquus rapitis superior und inferior; s.sp. = semispinalis capitis.

Der große Venenplexus der $V$. $v$ vertebralis und profunda cervicis liegt in dem Raum unterhalb des processus nastoideus bis hinab zum $M$. semi. spinalis (s.sp.), nach rorne bis zum longus capitis (l.c.). Sie setzen sich weiter fort in die Tiete uue bauen medial (unter) dem M. ubliquus capitis superior umfangreiche dichte Geflechte auf, die im vorliegenden l'räparat besoniers über dem Processus spinosus des Athits ausgebildet sind. In dem. Raum ror dem Longus capitis sind die l'Jexus erheblich geringer, (wichtig tür die Freilegung der Arterie zwischen dem ersten und zweiten foramen transicrsarium).

Fig. 7. Trockenpräparat. Erster bis dritler Italswirbel mil angrenzender Sichädelbasis, Felsenbein in der Verkürzung dirgestelle.

$P .:=$ Paukenhöhle.

A. a. vertebralis und carolis interna (ca.) injiziert. Letzlere tritt himier dem Processus styloideus in den Canalis caroticus ein.

Zwischen ersten und zweitem Wirbel beschreibt die $A$. vertebralis einen rechten Winkel, der nach vorne offen ist. Wird der Kopf nach links gredreht, so wandert der Querfortsatz des Atlas (l't.I.) nach vorne, der rechte Winkel wird zum spitzen (D r ün er); bei Drchung nach rechts wird er zum stumplen. In den Präparaten 3 und 5 ist durch das starke Hinüberlegen des Kopfes nacl: der gesunden Seite die Arterie gestreckt. Von der Konvexitä geht ein kleiner Asi ab (Ramus meningeus, vgl. Fig. 5), der durch das Foramen intervertebrale in den Wirbelkanal eintritt; aus dieser offnung tritt der $N$. cervicalis II herror (vgl. Fig. 5).

Oberhall des l'rocessus spinosus des Atlas (P.s.l.) macht die Arteric einen Bogen in horizontaler Ebene nach außen und hinten konvex, un durch die Membrana atlanto-occipitalis (M.o.) in den Wirbelkanal zu gelangen. Am hinteren Rande des Allaskörpers entspringt ein Ast für dic Meningen (A. meningea medid posterior intcrna).. Derartige Zwcige künnen bei stärlecrer lint. 
wirkfing intolge ihrer Anastomosen im Wirbelkanal slark bluten. (I. Fall $\mathrm{K}$ ü $\operatorname{tincr}$ )

Jaufige ist: tic Membran durch Knochen ersetzt, der $\Lambda$ tlas mit dem Occiput knöchern verbunden. Jann läuft die Arterie durch einen Knochenkana!.

Jer M. obliquus capitis inferior jst durch zwei rote Linien angedeutet.

$C$. = Gelenkspaltc zwischen crstem und zweitem IIalswirbel.

\section{Literaturverzeichnis.}

I. Dr un er, Ubet die Unterbindung der Arteria vertebralis. Tentralblatt f. Chir. 1917 , Nr. 30 .

2...-. Uber die chirurgische Anatomic der Arteria vertebralis. Beiträge $z$. klin. Chit, Bd. II2.

3. Goldam mer. Zitiert bei Hirtel I. c.

4. Härtel, Kriegsschußverletzungen des Halses. Ergcbnisse sler Chirurgie und Orthopädic, Bd. II.

5. H e y r owsky, Aneurysma spurium der Arteria vertebralis. Müncher med. Wochenschr. 1915, Nr. 2.

6. - Operation von selenen Aneurysmen. Gescllschaft der Ẍrate in Wien von 25. IV. 1919. Ref. Münchner med. Wochenschr. 1919, Nr. 22.

?. Ioseph, Aneurysma und Ligatur cler Aitcria vertebralis. Zentralbl. $\div$. Chiir. 1919, Nr. 23.

8. Ka usch, Dats Aneurysma der Arterat rertebralis und occipitale. Zent1albi. f. Chir. ig18, Ni. 47 .

9. Krausc, li, Dic operative Belıandlung der schweren Occipitalneuralgien. Verhandlungen der Deutschen Gesellschaft fuir Chirurgie I 899.

10. --. Kriegschirurgentagung Brüssel 1918 .

11. K i t 11 e $\mathrm{r}$, Ein typisches Verfahren zur Unterbinelung der Arteria vertebralis in der Suboccipitalregion. Zentralbl. f. Chir. 1917, Nr. 15.

12. -. Zur Frage der Vertebralisunterbindung. Zentralbl. f. Chir. 1917, Nir. 30.

I...-, Dic Verletzungen und traumatischen Aneurymen der Vertcbralgefäßo anı IIals: und ihre operative Behandlung. Bruns' Iieitr. z. Elin. Chir., Bi. 108.

14. -, Aneurysma der Vertebralis. Med Sektion der schles. (iesellachift für vaterländ. Kultur. 15. II. 1918.

15. -, Zur Vertcbralis.Diskusion. Zentall. f. Chir. 19ly. Nr. 25.

16. J. a ucuslein, Beitrag zun Aneurysma der Vertebralis. Zentralbl, f. Clin. 1918, Nr. 10.

17. I. 12 $z$, Jin Fall vou Aneurysma der Arteria vertebralis. Berliner klin. Wochenschr. 1917, $\mathrm{Nr}$. 19 .

18. Marmlil:, Gesellschaft der Ärzle in Wien. Wiener klin. Wochensthr. $1911, \mathrm{Nr} .19$.

19 Oljenjck, Uber dic Unterbindung: der Arteria vertebralis. Zentralbl. f. Cliit. 1917, Nr. 50 . 
Lur Technik der intraarteriellen Injektionen bej Gehirnerkrankungen usw. 235

20. Ostenberg, v. Aneurysma anteriorcnosum zwischen Carotis interna, Sinus vertebralis und Sinus transversus. Münchner med. Wochenschr. 191?, Nir. 7 .

21. Terthes, Zur Operation des Aneurysma der Arteria vertebralis. Zentralbl. f. Chis. $1918, \mathrm{Nr} .50$.

22. R i chter, lber Gefäßaneurysmen. Zitiert bei 1 l ärtel.

2.3. Sc h e m m e l, Beitrag zur B3ehandlung der Verletzungen der Arteria verte. bralis. Zentralbl. f. Chir. 19!s, Nr. 48.

24. Subotitch, Kriegschirurgische krfahrungen über traumatische Aneurysmen. Deutsche Zeitschr. f. Chir., Bd. 127. (Will bei Verletzung der cinen Seitc aut beiden Seiten unterbinden.)

25. I jetze. Aneurysma der Arteria vertebralis. Med. Sektion der schles. Gesellschatt füı vaterl. Kultur un Breslat. Sitzung vom 15. II. 1918. 


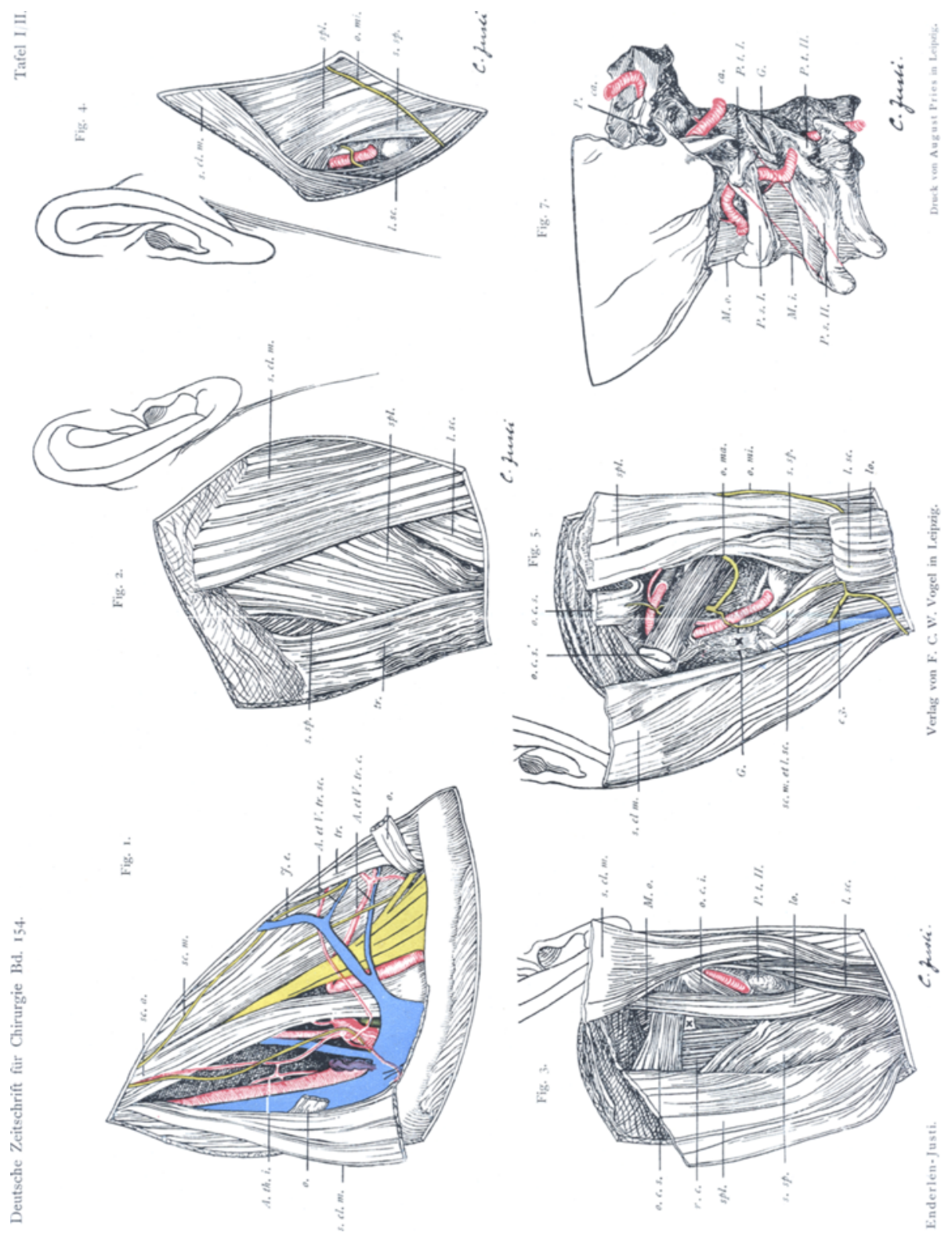




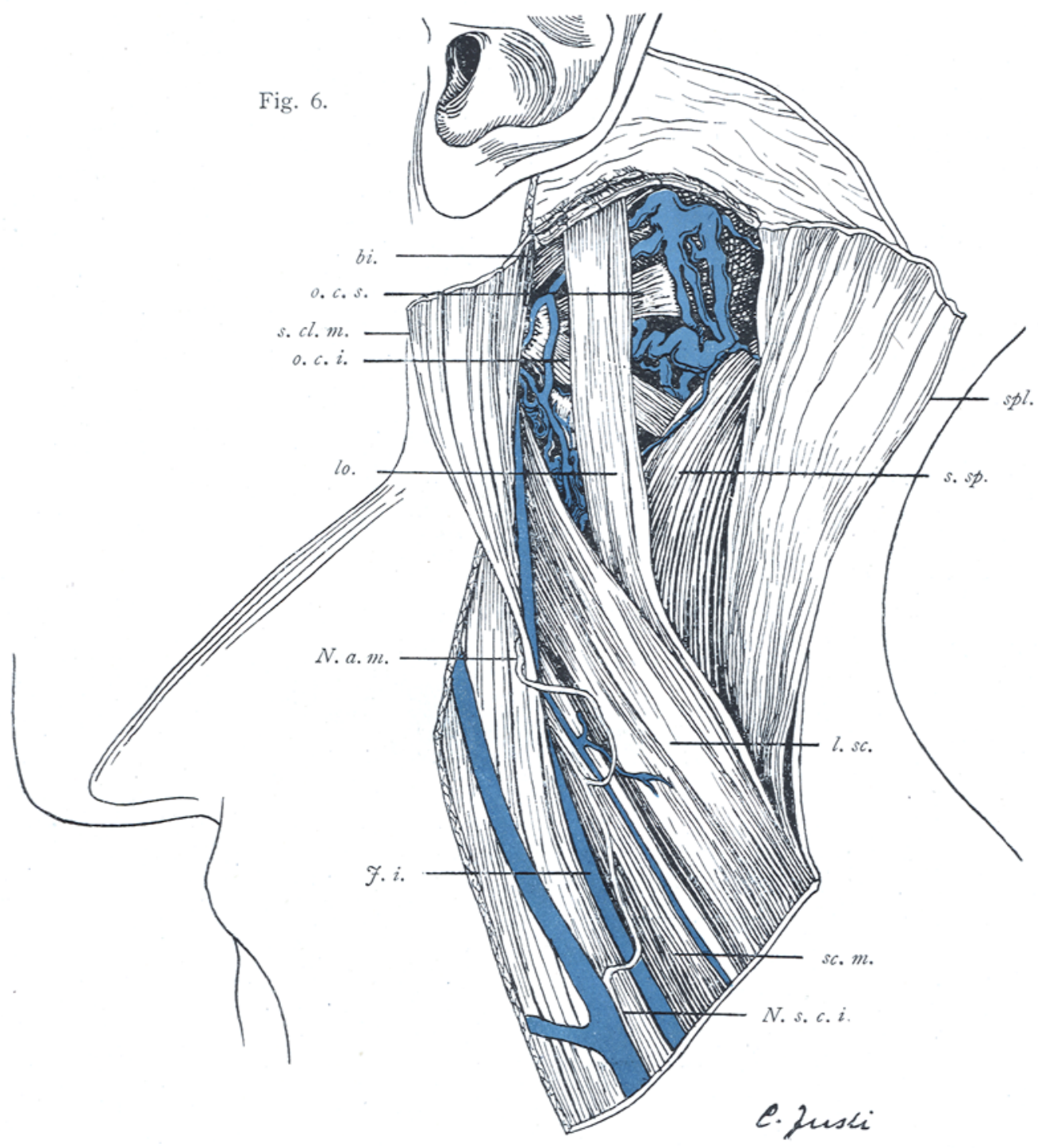

Enderlen-Justi.

Verlag von F. C. W. Vogel in Leipzig. 